\title{
Quantum Simulations of Hydrogen Bonding Effects in Glycerol Carbonate Electrolyte Solutions Supporting Information
}

\author{
Andrew E. Eisenhart*†, Thomas L. Beck* $\ddagger$ \\ *Department of Chemistry, University of Cincinnati, Cincinnati \\ E-mail: †eisenhaw@mail.uc.edu, $\ddagger$ beckt|@ucmail.uc.edu
}




\section{Pure GC}

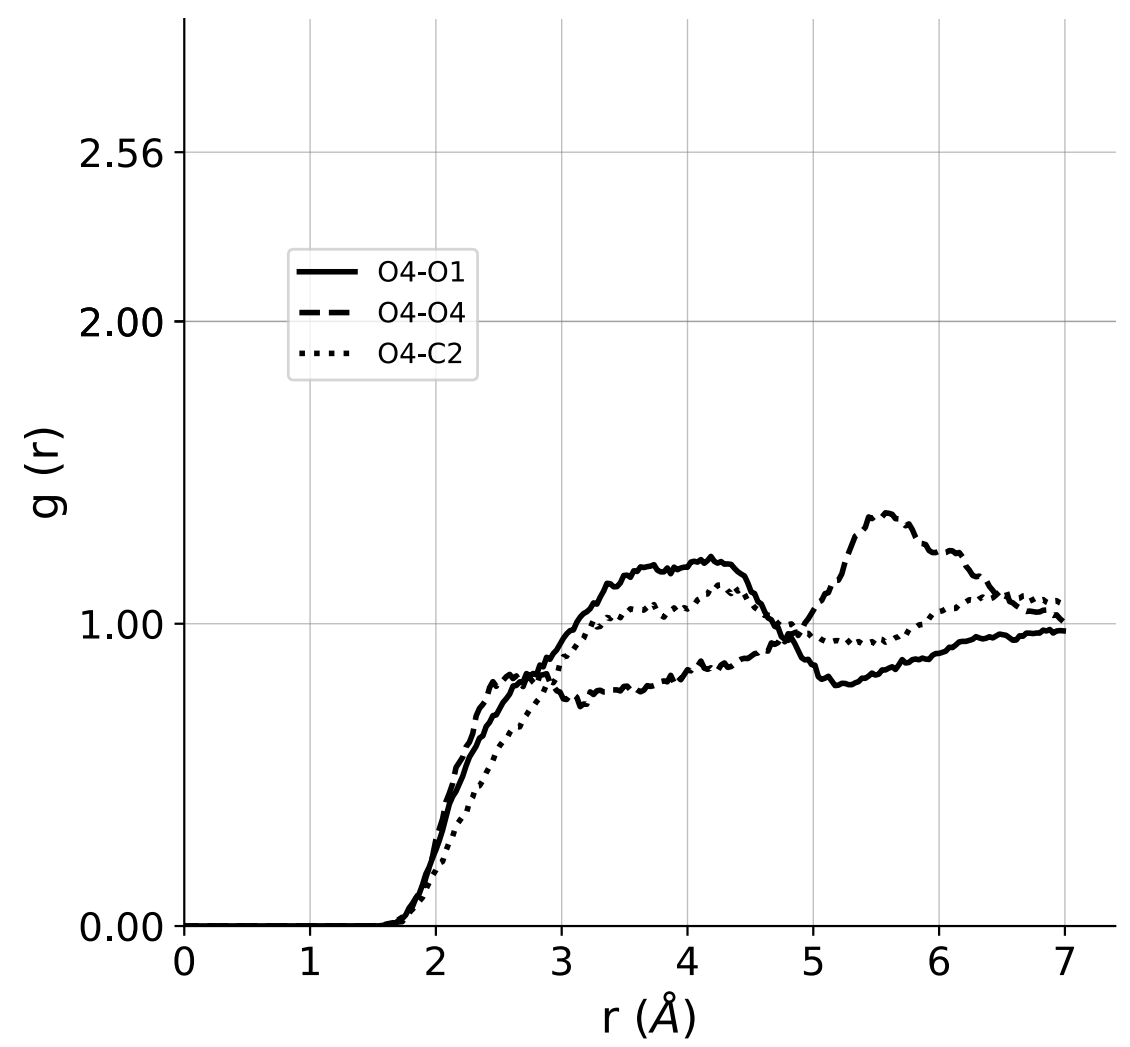

Figure S1: Radial distribution functions (RDFs) describing the O4-O4, O4-O1, and O4-C2 interactions. These RDFs show the ring-to-ring interactions in the pure GC system. All profiles generated from AIMD simulation data. 


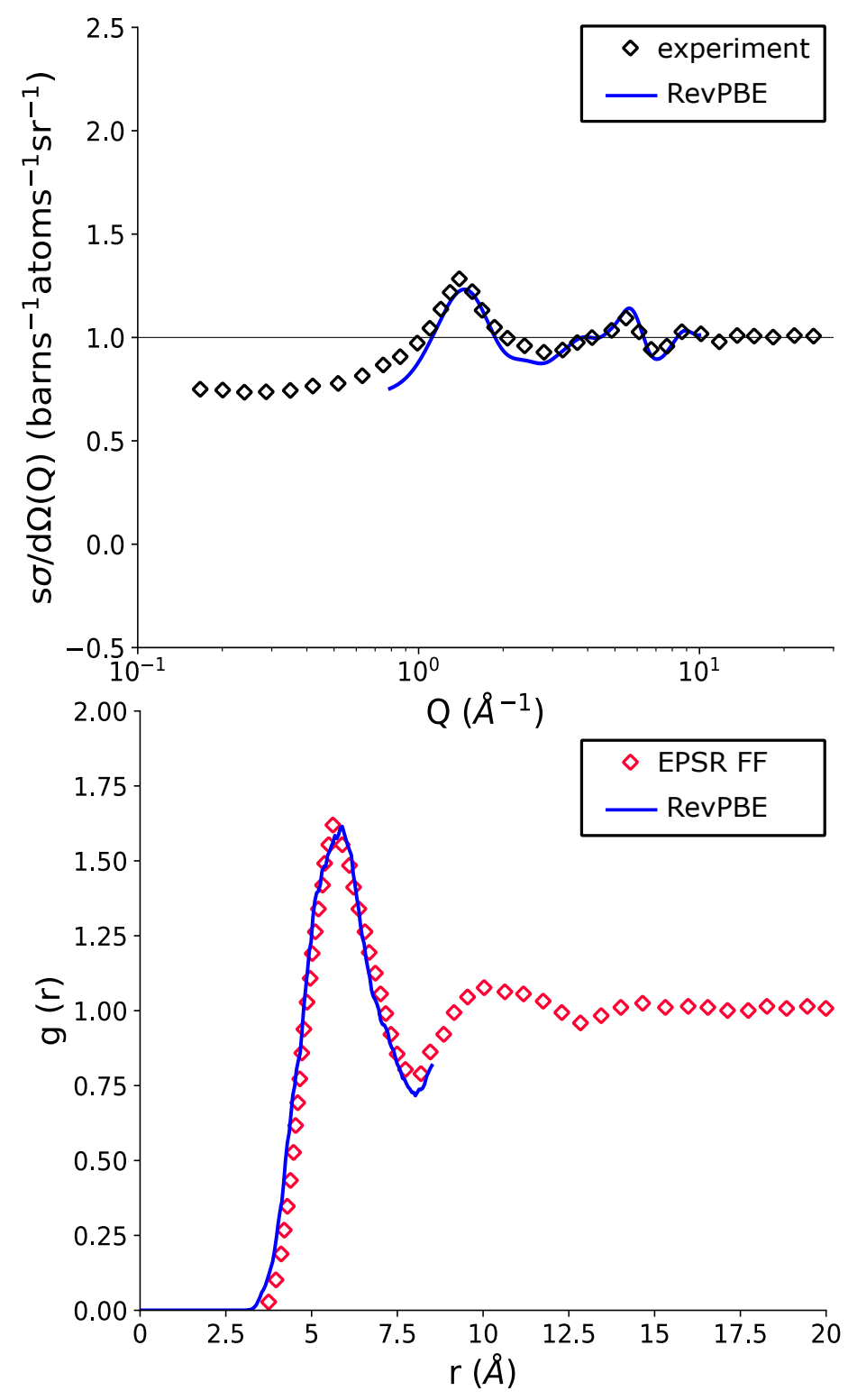

Figure S2: Top - Calculated (blue) and experimental (black diamonds) structure factors (top). Bottom - center-of-mass to center-of-mass RDFs from our AIMD simulations (blue) and the EPSRfit classical force field (red diamonds). Experimental structure factor data and classical center-ofmass to center-of-mass RDF recreated from Delavoux et al.[1] 


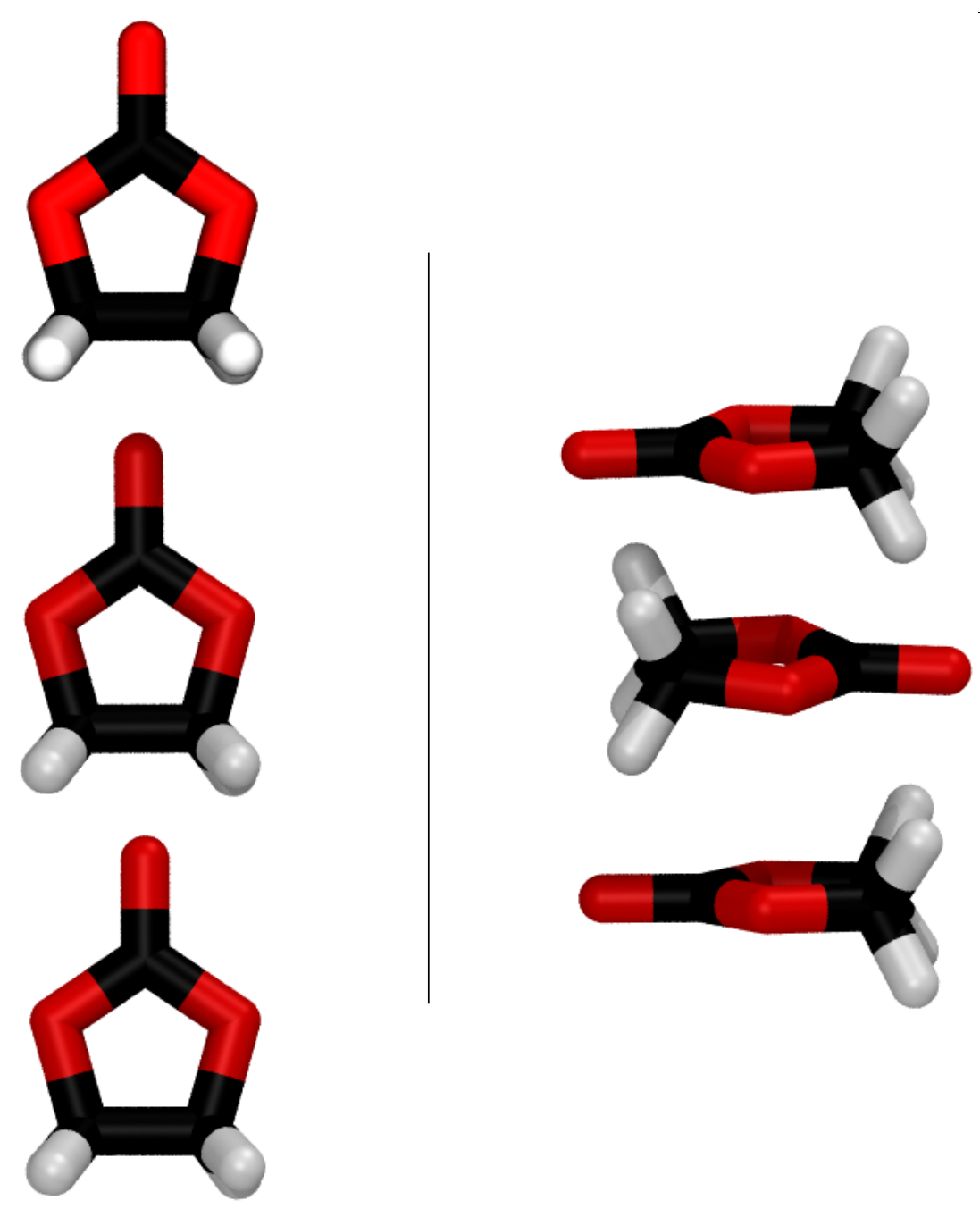

Figure S3: Schematic diagram showing ideal chain formation (left) and stacking (right) in ethylene carbonate. 


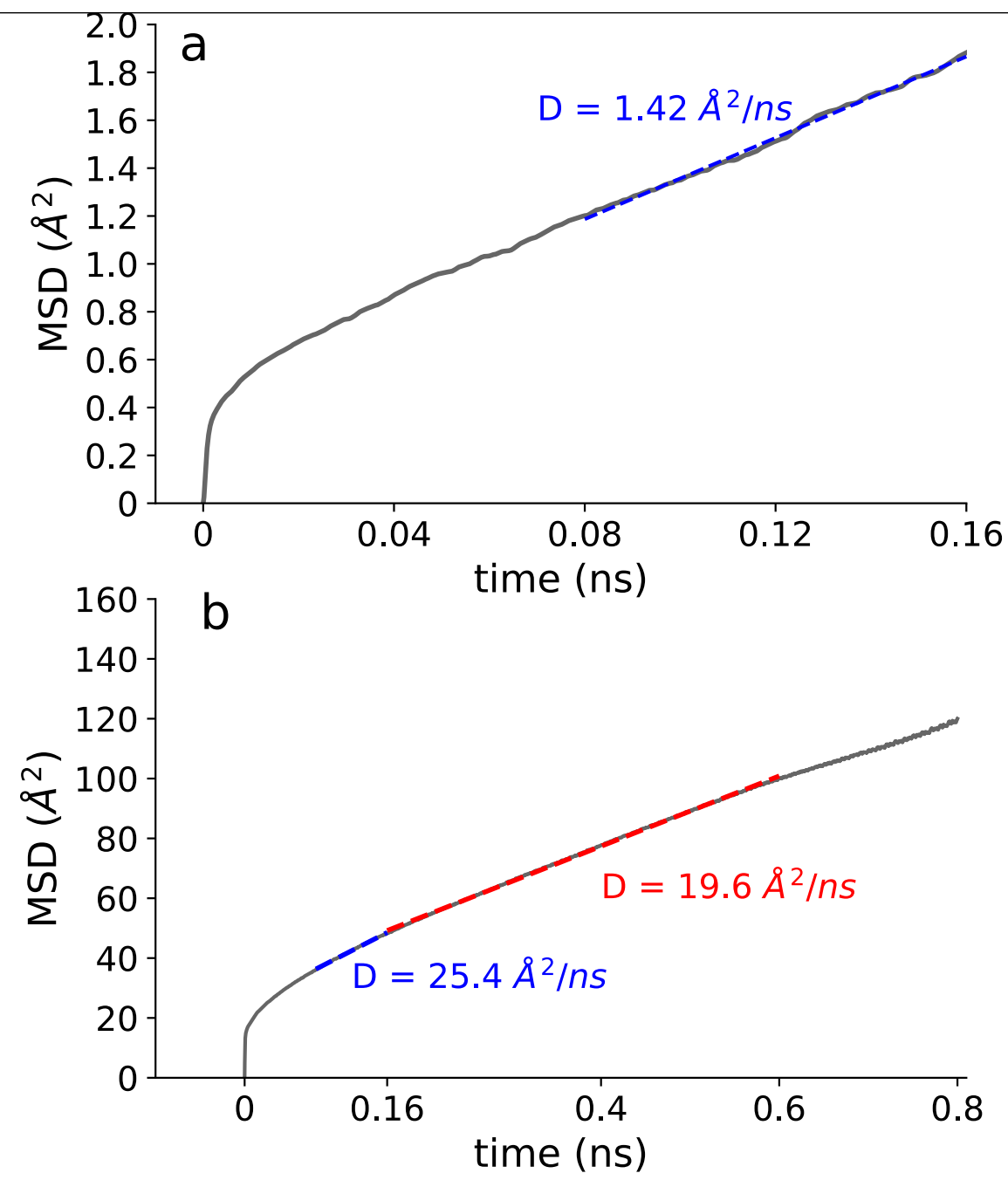

Figure S4: Calculated mean squared displacements for AIMD (a) and classical simulations (b). Linear regression fit trend-lines are displayed for short (blue, 0.08 to $0.16 \mathrm{~ns}$ ) and long (red, 0.16 to $0.6 \mathrm{~ns}$ ) timescales, along with correspondingly colored self diffusion coefficients.

The calculated MSDs show the linearity of the fit regions used to determine the diffusion coefficients obtained from the AIMD and classical simulations (Fig. S4 "a" and "b"). The AIMD time scale (blue) for fitting was validated by fitting a similar time scale in the classical simulation and comparing the resulting diffusion coefficient to that obtained over the longer fitting (red). The resulting values $19.6 A^{2} / n s$ (long window) and $25.4 A^{2} / n s$ (short window) differ by roughly $20 \%$, providing evidence that the AIMD simulations are long enough to calculate the self diffusion coefficients of pure GC to reasonable accuracy. 


\section{Scaled Charge Classical Simulations Compared to AIMD}

The following Figures (5-8) detail the results of our attempts to scale our classical model's (OPLSAA) partial charges to fit the ion-ion and ion-solvent RDFs generated by our AIMD simulations. Each graph focuses on one scaled model $(110,100,90,80$, or $70 \%$ of original charge) and surveys a variety of FK concentrations. Only the results which came closest to the AIMD simulation derived RDFs are shown here. Those include: 110 and $100 \%$ for the F to K RDFs (Fig. S5), 80\% for the fluoride and hydroxyl hydrogen RDFs (Fig. S6), and 70/80\% for the potassium to $\mathrm{O} 3$ and O4 RDFs (Fig. S7 and Fig. S8). Each classical system contains 2000 glycerol carbonate molecules and the labeled number of ion pairs.
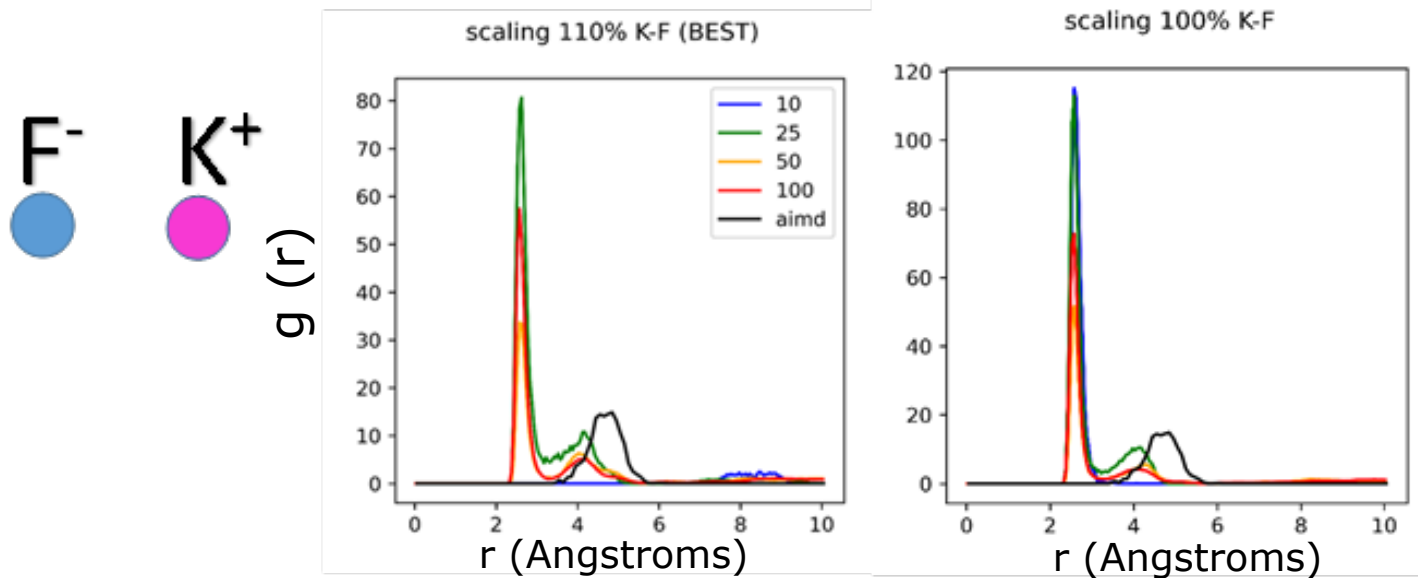

Figure S5: Left - The effect of electrolyte concentration (legend numbers correspond to the number of ion pairs in the system) using our $110 \%$ scaled classical OPLS-AA model for the ion association (K to F) RDFs compared to the AIMD results (black). This scaling came the closest to reproducing the AIMD results. Right - The same interactions with no scaling. 

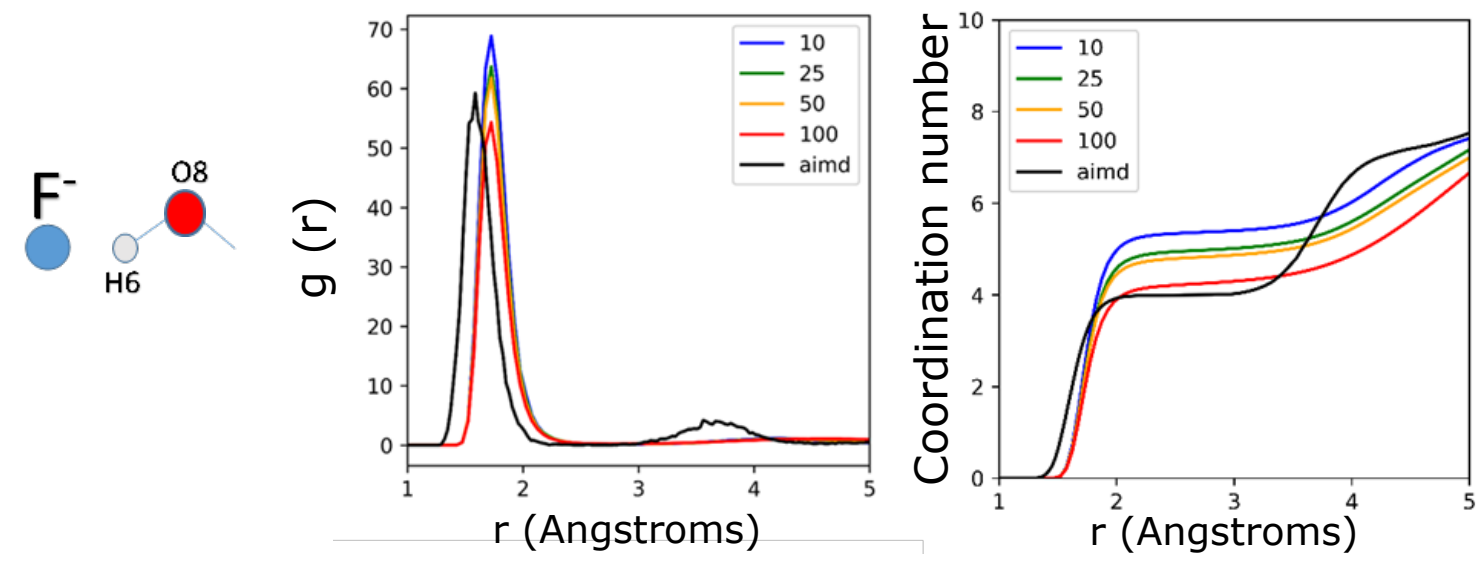

Figure S6: Left - The effect of electrolyte concentration using our $80 \%$ scaled OPLS-AA model on hydrogen bonding ( $\mathrm{F}$ to H6) RDFs compared to AIMD results. This scaling came the closest to reproducing the AIMD results. Right - The corresponding coordination numbers. Note the lack of secondary structure in the classical model at 3-4 $\AA$.
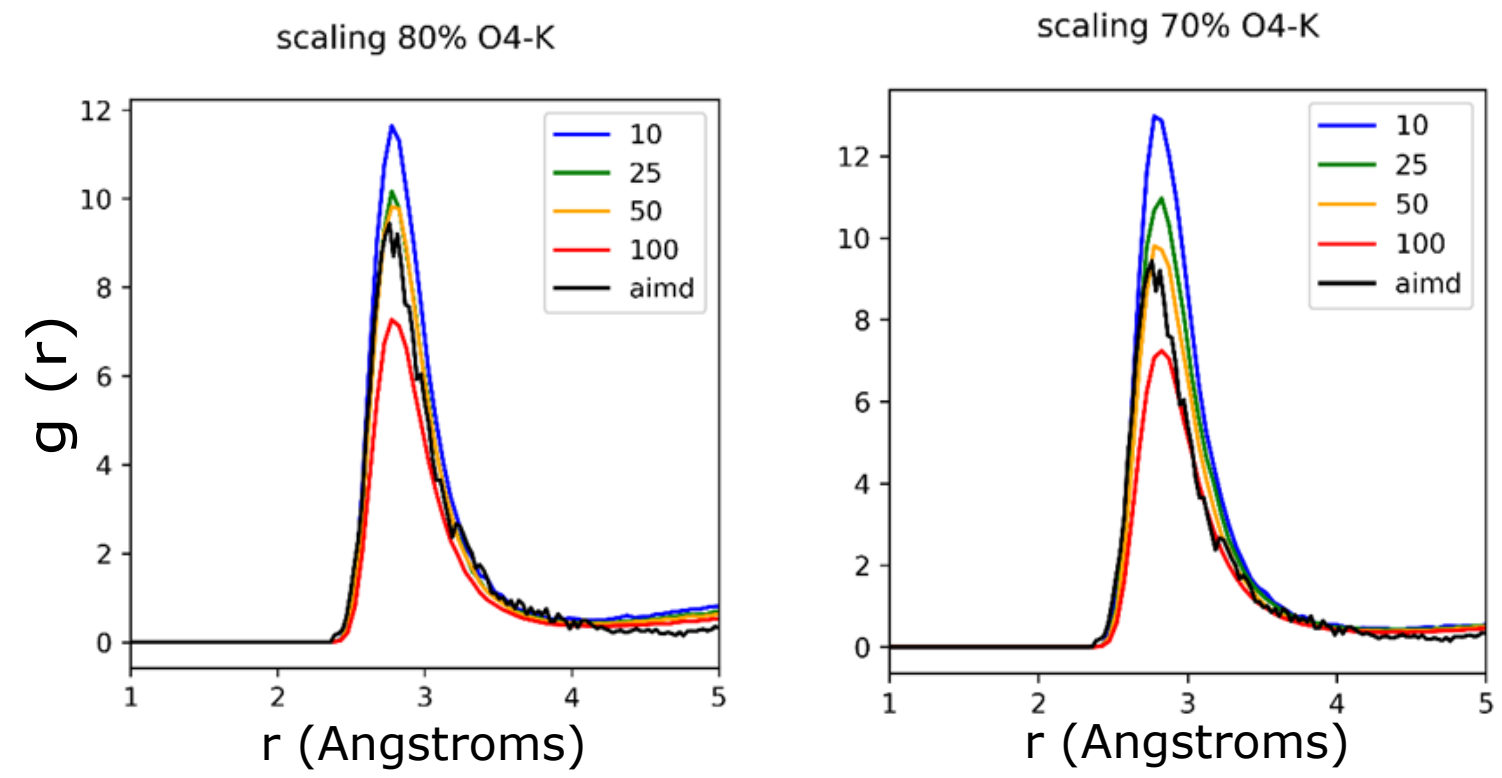

Figure S7: Left - The effect of electrolyte concentration using our 80\% scaled OPLS-AA model on carbonyl bonding (K to O4) RDFs compared to AIMD results. Right - The effect of electrolyte concentration using our 70\% scaled OPLS-AA model on carbonyl bonding (K to O4) RDFs compared to AIMD results. 

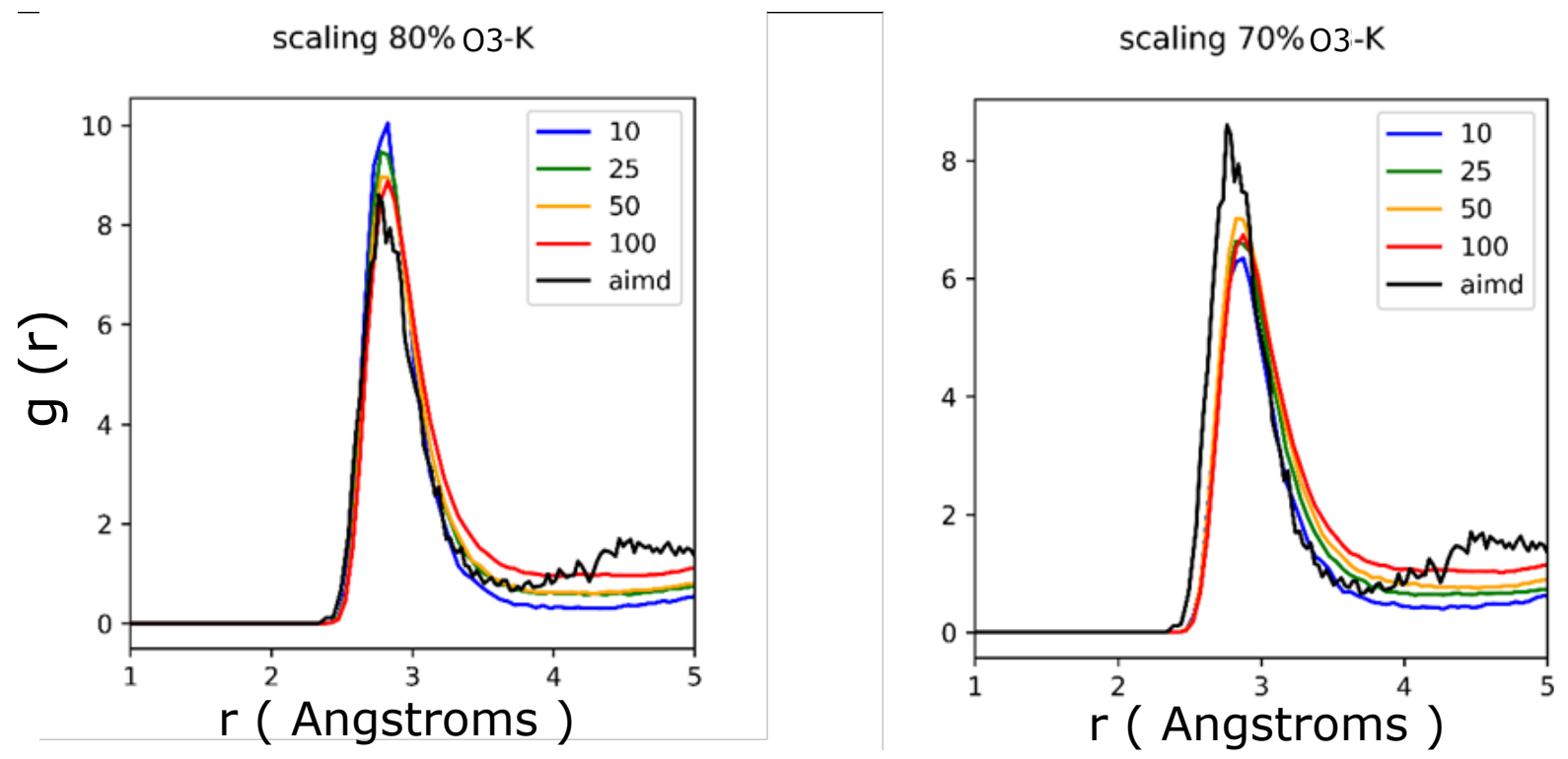

Figure S8: Left - The effect of electrolyte concentration using our 80\% scaled OPLS-AA model on carbonyl bonding (K to O3) RDFs compared to AIMD results. Right - The effect of electrolyte concentration using our 70\% scaled OPLS-AA model on carbonyl bonding ( $\mathrm{K}$ to O3) RDFs compared to AIMD results.

\section{Pure GC charge transfer}

To further characterize the hydrogen bonding present in the pure GC system we have calculated and analyzed the electron density and atomic charge transfer between GC dimers using the ORCA quantum chemistry program and the Bader charge partitioning method. Fig. S9 illustrates the qualitative effect of GC dimer association on the electron density near the binding sites. 

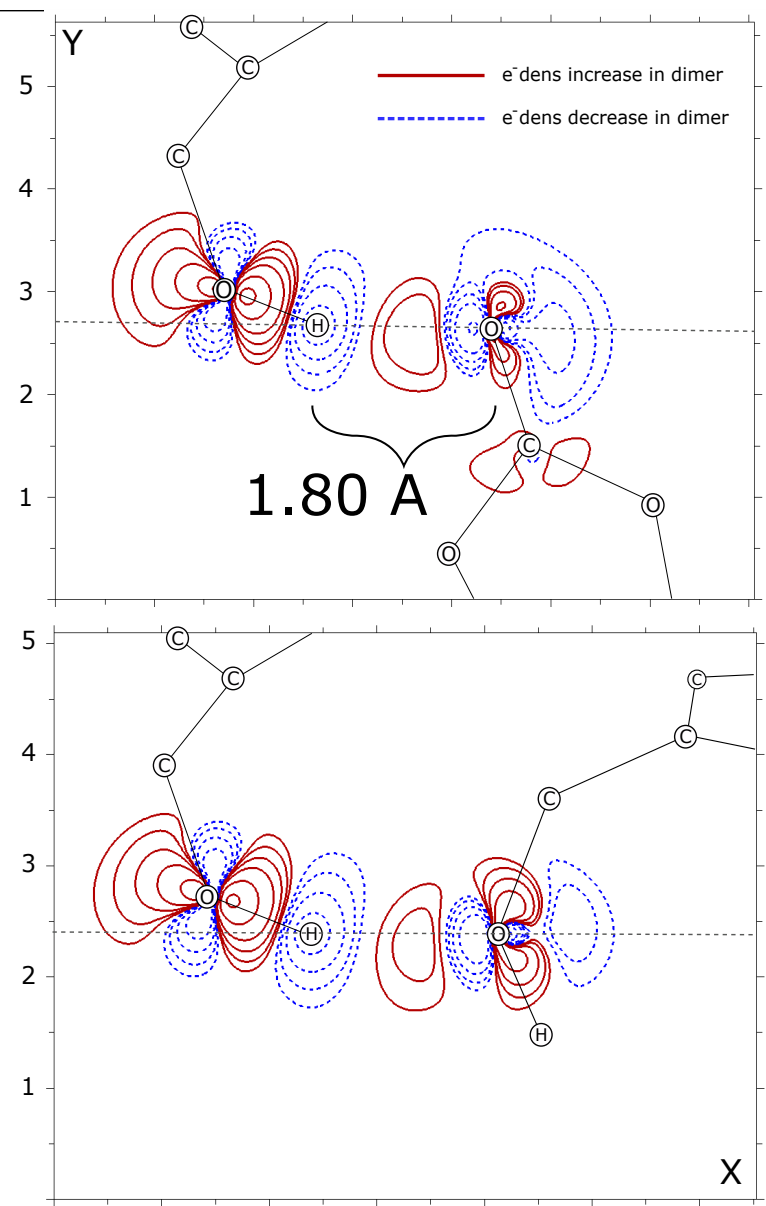

Figure S9: The $e^{-}$density change as a result of GC-GC hydrogen bonding: carbonyl-to-tail (top), and tail-to-tail (bottom).

The $\mathrm{e}^{-}$density changes in Fig. S9 are calculated in a plane that intersects the binding atoms in each dimer. The $\mathrm{e}^{-}$density increase (red - solid) or decrease (blue - dotted) compared to isolated GC molecules is shown along this plane. Atom site representations were added into the image for clarity, but only the atoms involved in the hydrogen bonding (hydroxyl oxygen and hydrogen, carbonyl oxygen) are in the same plane as the calculated electron density. Both $\mathrm{e}^{-}$density images show the polarization of the hydroxyl tails when participating in hydrogen bonding. This can be seen in the decreasing density around the hydrogen and increasing density around the oxygen when bound to either the carbonyl or another hydroxyl tail. There is a clear multipolar character to the charge rearrangements.

In Fig. S10, the atomic partial charge distributions for each of the atoms that directly participate in hydrogen bonding have been calculated. Beginning with the tail-to-tail interactions (Fig. S10 "a" \& 
"b"), we see that the oxygen (OA) interacting with the hydrogen sees little change in its charge distribution, but the electron density around the oxygen $(\mathrm{OB})$ that is attached to the associating hydrogen is shifted towards the left (more negative partial charge or higher $\mathrm{e}^{-}$density). The two hydrogens in this interaction are both polarized to the right (slightly more positive partial charge or lower $\mathrm{e}^{-}$density), with the hydrogen (HB) sandwiched between the two oxygens more positively charged than the other.

The carbonyl-to-tail hydrogen bonding conformation displays similar charge transfer behavior, with the hydroxyl oxygen (OB) not experiencing much polarization. The carbonyl oxygen is more negatively charged than its monomer counterpart (M-OX). The hydrogen (HB) involved in this bond exhibits significant polarization compared to its monomer counterpart, displaying the largest difference between monomer and dimer in terms of charge transfer in either of the conformations.

Summary of charge transfer data in pure GC: The site-specific polarization that is evidenced in Fig. S10 implies the pure GC system is difficult to model with a classical point charge representation. Scaling the atomic charges to those found in either of these dimer configurations would lead to the incorrect charges for the other interaction. This would lead to one interaction being either over or under-estimated. The addition of site-specific polarization is necessary to mimic the changing charge on the specific atoms involved in the hydrogen bonding. Due to the seeming reliance of these models on the accuracy of the hydrogen bonding interaction we believe that Drude or point-polarizable point-dipole models with polarizable sites centered on the carbonyl and hydroxyl tail of GC may improve agreement with the AIMD simulations relative to a fixed charge model. Based on the complexity apparent in Fig. S9, it is apparent that a complicated array of such oscillators may be required to accurately reproduce the interactions, however. 

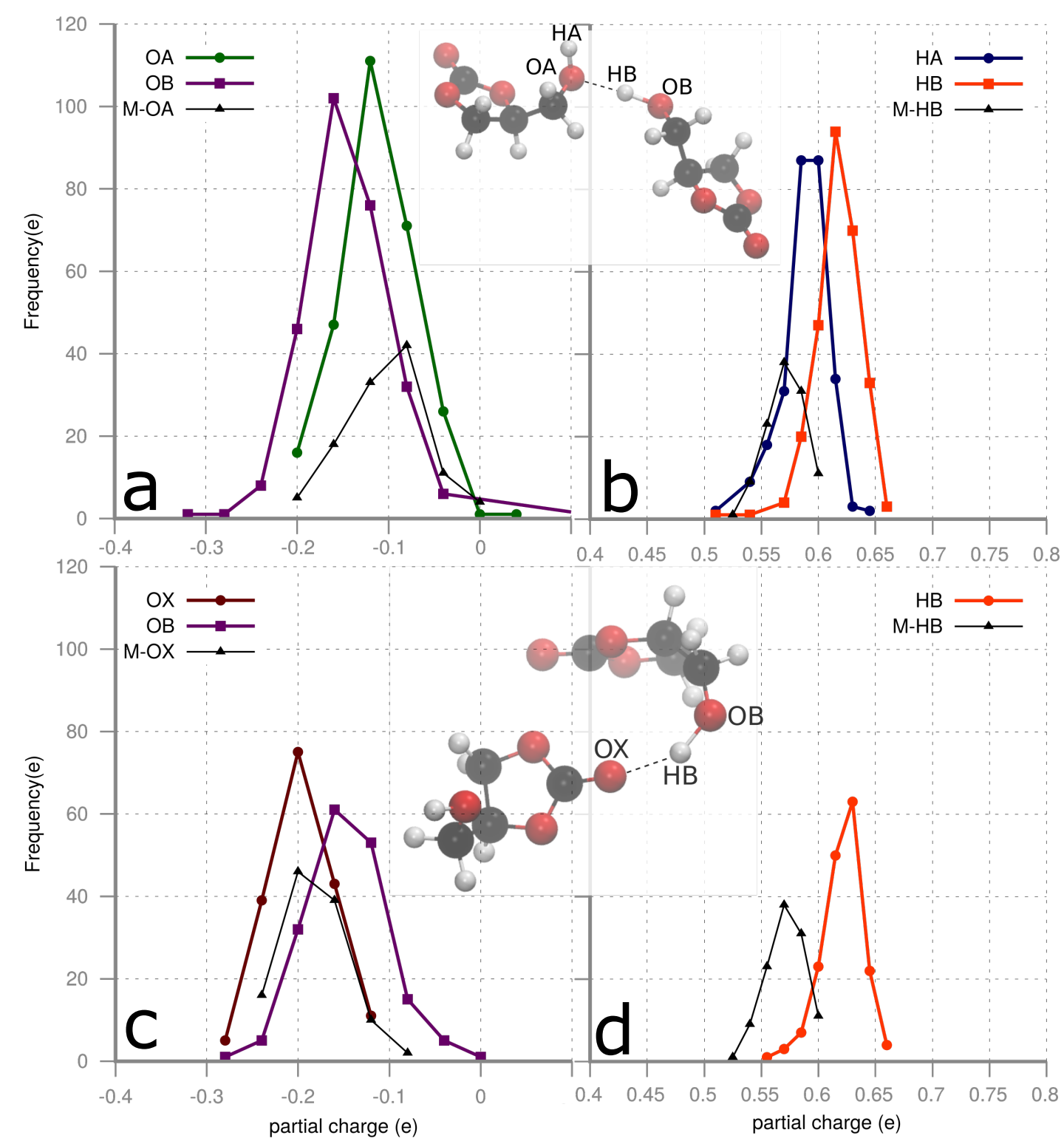

Figure S10: The Bader atomic charge for atoms involved in the two modes of GC-GC association, tail-to-tail (a, b) and tail to carbonyl (c, d). The "M" labels indicate the monomer counterpart to the observed atoms. Graph "a" displays the partial charge distributions for the oxygens involved in the tail-to-tail interaction. Graph "b" displays the partial charge distributions for the hydrogens of the same interaction. Graph "c" details the partial charge of the oxygens in the carbonyl-to-tail interaction, and graph "d" follows the partial charge of the lone hydrogen involved in that same interaction. 


\section{GC electrolyte solution charge transfer}

The partial charge distributions in Figs. S11, S12, and S13 display the charge transfer occurring between the three ions studied in our work and their first solvation shells. Configurations used for these calculations were obtained from AIMD simulations of the respective ion solvated in 27 GC molecules (without a counterion). A distance cutoff specific to each system was used to isolate the first solvation shell around each ion $(2.5 \AA$ for fluoride, $3 \AA$ for chloride, and $4 \AA$ for potassium). These distances were determined from the first valley in the relevant RDFs (main text Fig. 9 \& Fig. 10). 100 of these configurations for each system were used to generate the distributions.

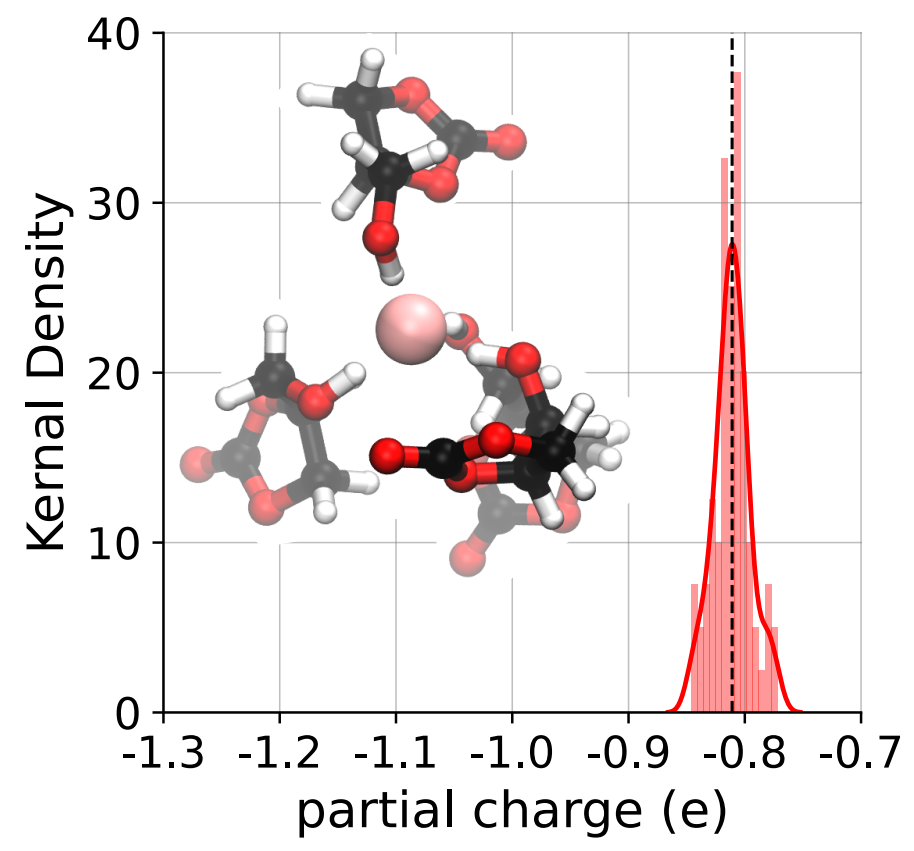

Figure S11: The partial charge distribution of a fluoride ion in a 4-GC cluster. The mean of the distribution, marked by the vertical dashed line, is -0.811 . This distribution has a standard deviation of 0.015 .

The fluoride ions in these clusters and in the KF electrolyte solutions are solvated by an average of 4 GC molecules arranged in a geometry close to tetrahedral (see main text Fig. 11). We find that the fluoride ion donates on average -0.189 e of its charge to the surrounding 4 GC molecules, retaining a mean partial charge of -0.811 e. This value is very close to the charge transfer undergone by the fluoride ion inside the E148-P binding site found in our previous work on the EcCLC chloride transporter protein Table 2 of Ref.[2]. 


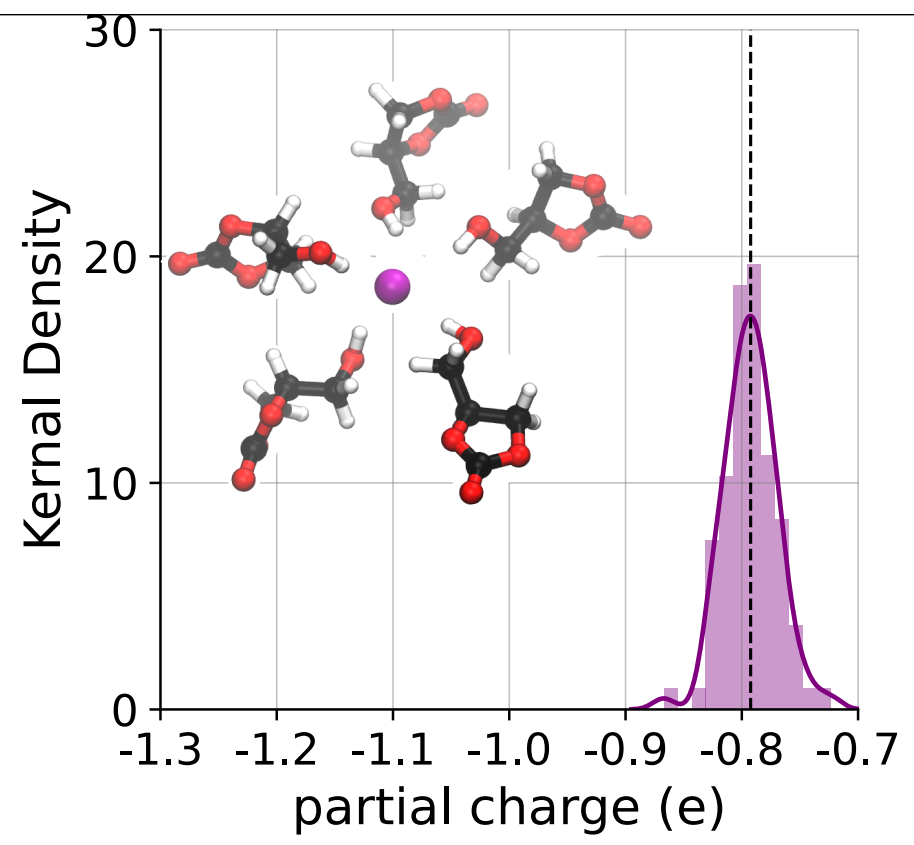

Figure S12: The partial charge distribution of a $\mathrm{Cl}^{-}$ion in a $5 \mathrm{GC}$ cluster. The mean of the distribution, marked by the vertical dashed line, is -0.792 . This distribution has a standard deviation of 0.023 .

The chloride ion by itself is solvated differently by GC than when paired with potassium. Here we see that an average of 5 GC molecules bind directly to the anion through their hydroxyl tails. This is 2.5 more GC molecules on average than is seen binding to the chloride ion when $\mathrm{K}^{+}$is present. Similar to fluoride, the chloride anion also donates a portion of its charge to the first solvation shell $(-0.208 \mathrm{e}$ on average). This leaves the chloride ion with a partial charge of $-0.792 \mathrm{e}$. Thus, the fluoride and chloride ions are relatively close in terms of partial charge when solvated by GC. 

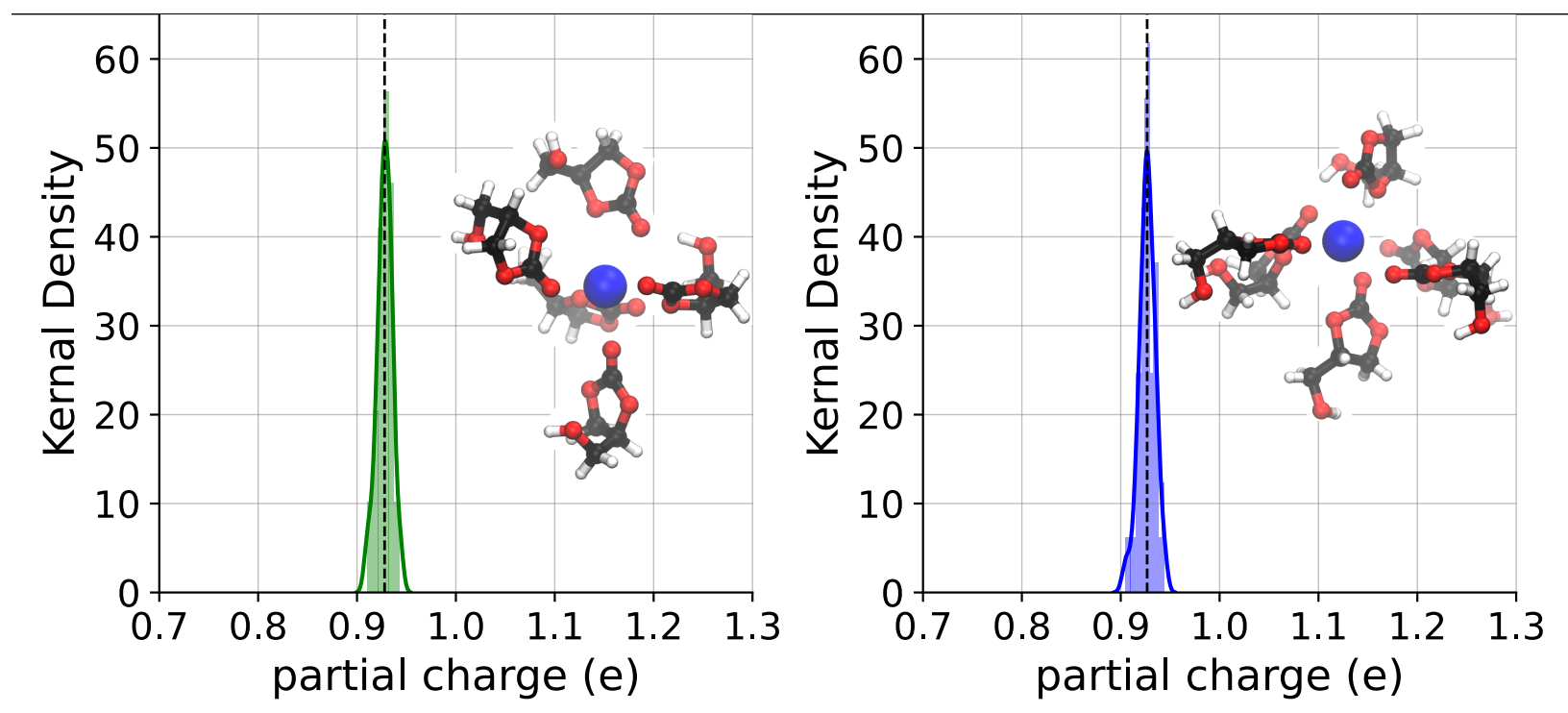

Figure S13: The partial charge distribution of a potassium ion in a 5 (left) or 6 (right) GC cluster. The means of the distributions, marked by the vertical dashed lines, are +0.928 and +0.927 , and the distributions have standard deviations of 0.007 and 0.008 .

In our single solvated potassium ion system, we see two different solvation shells that can form around the cation. The first has 5 GC molecules in its first shell. In this case, the potassium ion carries a mean partial charge of +0.928 e. The second type of cluster has a sixth GC molecule that enters the first solvation shell. Here the mean partial charge on the potassium ion is very slightly reduced to +0.927 e.

\begin{tabular}{lll} 
Cluster & $\bar{q}(\mathrm{e})$ & $\sigma(\mathrm{e})$ \\
\hline $\mathrm{GC}_{4}[\mathrm{~F}-]$ & -0.811 & 0.015 \\
$\mathrm{GC}_{5}[\mathrm{Cl}-]$ & -0.792 & 0.023 \\
$\mathrm{GC}_{5}[\mathrm{~K}+]$ & +0.928 & 0.007 \\
$\mathrm{GC}_{6}[\mathrm{~K}+]$ & +0.927 & 0.008
\end{tabular}

Table S1: Ionic partial charges

Summary of charge transfer data for the ion/GC systems: Comparing all of our ionic partial charges in Table S1, we can see that the mean partial charges of the fluoride and chloride ions are very similar when solvated by GC. The "hard" nature of fluoride's charge seems to be balanced out by its formation of more "chemical" bonds with the hydroxyl hydrogens of GC. This enhances the energetics of the binding of GC to fluoride relative to the softer (more polarizable) chloride ion. Both fluoride and chloride anions 
share almost twice as much of their charge with their first solvation shell when compared to the charge acquired by the potassium cation. This highlights the difference in interactions of the anions with GC compared to the cation. This may help to explain why the identity of the anion is so important in determining the macroscopic properties of the GC electrolyte solutions.

\section{References}

[1] Yoan M Delavoux, Mark Gilmore, Martin P Atkins, Małgorzata Swadźba-Kwaśny, and John D Holbrey. Intermolecular structure and hydrogen-bonding in liquid 1, 2-propylene carbonate and 1, 2-glycerol carbonate determined by neutron scattering. Physical Chemistry Chemical Physics, 19(4):2867-2876, 2017.

[2] Zhihong Chen and Thomas L Beck. Free energies of ion binding in the bacterial clc-ec1 chloride transporter with implications for the transport mechanism and selectivity. The Journal of Physical Chemistry B, 120(12):3129-3139, 2016. 